\title{
Analysis and Experimental Validation of a Type of Three-Microstrip Directional Coupler
}

\author{
Fátima Masot, Francisco Medina, and Manuel Horno, Member, IEEE
}

\begin{abstract}
This paper presents an efficient analysis of a semi reentrant microstrip coupler section. The structure provides tight coupling while maintaining all connections at the same plane. The scattering parameters of this coupler were obtained in the past using the even-odd mode theory. This paper shows that three, rather than two, fundamental modes should be considered in a general situation, since the even-odd mode theory is inaccurate in certain cases. The coupler quasi-TEM parameters are computed by using an enhanced version of the spectral domain technique. Thus, the synthesis of the coupler is carried out in seconds using a personal computer. A rigorous full-wave analysis has also been implemented to ensure that dispersion is negligible. Experimental validation of theoretical conclusions is included.
\end{abstract}

\section{INTRODUCTION}

$\mathbf{H}$ IGH coupling is not achievable in conventional edge coupled microstrip couplers due to the need for impracticable inter line spacing. Among the solutions existing for this problem [1], the semi re-entrant microstrip section shown in Fig. 1 is a relatively simple and feasible choice. The influence of an additional floating strip printed at the other side of the substrate drastically increases the coupling between the two coplanar strips [2]. Note that this structure is suitable for interconnection in an (M)MIC frame since all connections are located on the same plane. This structure has already been analyzed by means of approximate [2] and field-theory based quasi-TEM techniques (spectral domain [3] and boundary division method [4]). In these papers, the symmetry of the structure reduces the three-conductor problem to an equivalent two-conductor one. Thus, the effect of the floating conductor is to modify the even, $C_{e}$, and odd, $C_{o}$, mode capacitances of the edge-coupled strips. This assumption leads to correct results in certain practical situations, as shown by experimental evidence in [2]-[4]. Nevertheless, it could eventually yield inaccurate results in other cases. This is because the actual structure supports three (instead of two) quasi-TEM-type orthogonal modes. The characteristic parameters of these modes must be computed by solving the pertinent eigenvalue problems [5], [6]. One of these modes is the so-called odd mode in [3], [4]. However, there are two different even modes-with different propagation constants, line impedances and mode numbersthat differ from the conventional even mode in [3], [4]. In this paper, we refine the theory in [3], [4] to account for this. In

Manuscript received July 19,1993; revised October 25, 1993. This work was supported in part by the DGICYT, Spain, Project No. TIC91-1018.

The authors are with the Grupo de Microondas, Departamento de Electrónica y Electromagnetismo, Facultad de Física, Universidad de Sevilla, Avenida Reina Mercedes s/n, 41012 Sevilla, Spain.

IEEE Log Number 9404099.

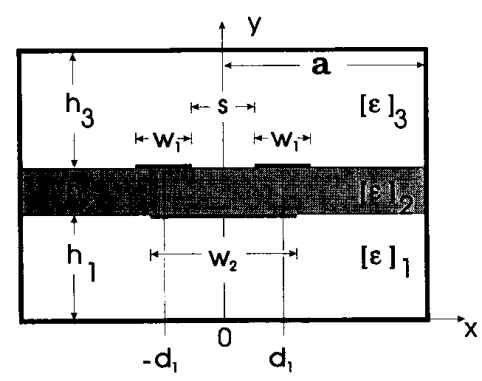

Fig. 1. Cross section of the semi reentrant three-microstrip coupler analyzed in this work. The three dielectric layers may be iso/anisotropic materials or air.

addition, we study the conditions under which the conventional even-odd mode theory can be applied confidently.

On the other hand, the analysis of the electrical response of the coupler system requires the previous knowledge of the modal parameters. These can be obtained from either a quasi-TEM or full-wave analysis. The latter is computationally intensive and should be avoided if dispersion is expected to be negligible. Under quasi-TEM operation the line parameters can be fully derived from the per unit length (p.u.l.) capacitance, $\overline{\bar{C}}$, and inductance, $\overline{\bar{L}}$, matrices of the structure. Quick, accurate, and reliable computation of these matrices is desirable if we want to use an iterative optimization scheme to design the coupler. An appropriate general method to analyze our structure is, for instance, the variational spectral domain method [7]. However, the straightforward formulation of that technique is still computationally intensive for our purpose. Fortunately, we can use the efficient scheme and formulae reported in [8] to carry out intermediate computations by properly exploiting the symmetry of the structure. Thus, we drastically speed up the computation in such a way that the analysis program codes become suitable for automatic design. Once the primary $\overline{\bar{C}}$ and $\overline{\bar{L}}$ matrices are known, the secondary line parameters are easily obtained [5]. Then, the scattering parameters - or the impedance matrix - of the six-port circuit are computed by following, for instance, the theory in [5] or [6]. Finally, the scattering parameters of the four-port coupler are derived from those by imposing the proper termination conditions at the ports. The scattering parameters provided by the more conventional even-odd mode analysis have also been computed for comparison purposes. We have made some experiments to determine the validity of the theories in this paper. 
Note that we use the quasi-TEM model for design purposes, since it is much simpler than the full-wave one. However, since the structure is not homogeneous (and consequently dispersive in nature) a full-wave analysis has also been performed to make sure of dispersion is negligible. The full-wave analysis is carried out by means of the spectral domain approach enhanced with the same techniques used in quasi-TEM computations. The results obtained with this approach show that quasi-TEM data are good enough for the dimensions and frequencies considered in this paper. In other case (higher frequencies), full-wave data should be used to design the coupler. In this case the process obviously becomes much more time consuming.

\section{ANAlysis of the Coupled Section}

The structure in Fig. 1 may be analyzed as a backward wave directional coupler of length " $l$ ", by following two different approaches. The first uses the approximate even-odd mode model; the second considers the existence of three distinct quasi-TEM modes.

\section{A. Even-Odd Mode Approach}

The starting hypothesis is that the basic coupler features can be derived from a quasi-static analysis assuming that the total charge on the floating conductor is zero [3], [4]. Thus, the complete $3 \times 3$ capacitance matrix, $\overline{\bar{C}}$, reduces to a $2 \times 2$ one whose eigenvalues are the even $\left(C_{e}\right)$ and odd $\left(C_{o}\right)$ mode capacitances. They are related to the even and odd charge excitations of the two hot conductors: $(+1,0,+1),(+1,0,-1)$. These pseudo eigenvectors generate a bidimensional space, in such a manner that the three-conductor problem is treated as a two-conductor one. The same arguments apply to the capacitance matrix in vacuum, $\overline{\bar{C}}_{v}$. If this model is adopted, the scattering parameters of the coupler are easily computed using the well known even-odd mode theory [9].

We have implemented this model to compare with the more rigorous three-mode model. Efficient computation of the mode capacitances will be discussed later. Our program codes have been checked against the results reported in [3], [4]. We found very good agreement with the theoretical and experimental data reported there. The good accordance between theoretical and experimental data in these cases shows that the even-odd mode theory works sufficiently well for at least some practical situations. However, we found significant discrepancies between our simulation and experimental data for other coupler realizations. We explain this disagreement in the light of the three-mode model. The question is under what conditions is the theory in this section applicable or not? We will try to answer this question in a later section.

\section{B. The Three-Mode Approach}

In the frame of quasi-TEM theory, the three-conductor symmetrical system under study supports three quasi-TEMtype fundamental modes. Each has its set of characteristic parameters (effective dielectric constants, modal line impedances, and modal numbers) which are computed solving the following eigenvalue problems [10]:

$$
\begin{array}{r}
{\left[\overline{\bar{L}} \cdot \overline{\bar{C}}-\frac{\epsilon_{p}}{c^{2}} \overline{\bar{U}}\right] \cdot \bar{V}_{p}=0} \\
{\left[\overline{\bar{C}} \cdot \overline{\bar{L}}-\frac{\epsilon_{p}}{c^{2}} \overline{\bar{U}}\right] \cdot \bar{I}_{p}=0}
\end{array}
$$

where the subscript $p=a, b, c$ stands for the mode; $\epsilon_{p}$ stands for the normalized modal effective dielectric constant; $\bar{V}_{p}$ and $\bar{I}_{p}$, the voltage and current eigenvectors, and $\overline{\bar{U}}$ is the $3 \times 3$ identity matrix. Closed-form expressions for the modal parameters of the symmetrical three-line system studied here were reported in [5] and have been included in the Appendix.

From the perspective of circuit theory, the three-strip structure may be considered a six port lossless reciprocal network (see Fig. 2(a)). Once the modal parameters and the coupling length are known, any of the approaches reported in [5] or [6] may be followed to evaluate the electrical response of the network (i.e. the impedance or the scattering matrix). If the impedance matrix approach is chosen, we can write for the six port circuit

$$
\bar{V}_{6}=\overline{\bar{Z}}_{6} \cdot \bar{I}_{6}
$$

where $\overline{\bar{Z}}_{6}$ is the $6 \times 6$ impedance matrix of the network and $\bar{V}_{6}$ and $\bar{I}_{6}$, the voltages and currents at the six ports. $\overline{\bar{Z}}_{6}$ is computed from the modal parameters and coupling length $(l)$ as reported in [5]. If the structure works as a directional coupler, we must obtain the impedance matrix of the corresponding four-port network, $\overline{\bar{Z}}_{4}$. Grouping together the elements corresponding to the four-port subnetwork-ports 1-2-3-4, in Fig. 2(a) - and the elements corresponding to the remaining two-port subnetwork - ports 5-6 -, we can write

$$
\begin{aligned}
& \bar{V}_{4}=\overline{\bar{Z}}_{4} \cdot \bar{I}_{4}+\overline{\bar{Z}}_{42} \cdot \bar{I}_{2} \\
& \bar{V}_{2}=\overline{\bar{Z}}_{24} \cdot \bar{I}_{4}+\overline{\bar{Z}}_{2} \cdot \bar{I}_{2}
\end{aligned}
$$

where the voltage and current vectors at the ports of the four-port and two-port subnetworks are distinguished with the subscripts 4 and 2 , respectively. The same convention,-indicating matrix dimensions-has been applied to the square self-impedance matrices, $\overline{\bar{Z}}_{4}$ and $\overline{\bar{Z}}_{2}$. The $\overline{\bar{Z}}_{42}$ and $\overline{\bar{Z}}_{24}$ are $4 \times 2$ and $2 \times 4$ mutual impedance matrices relating the electrical quantities at the ports of the two defined subnetworks. We may now assume that a perfect open end condition stands at the ends of the floating conductor $\left(\bar{I}_{2}=0\right)$ (Fig. 2(b)). This condition leads directly to the knowledge of the four-port impedance matrix, $\overline{\bar{Z}}_{4}$. Under this assumption, the voltage/current relationship for the four-port subnetwork is completely uncoupled from the voltage values at the two-port one.

Nonideal open-end effects could eventually play an important role in the electrical response of the network. This effect can be taken into account by including the open-end capacitance $\left(C_{\mathrm{op}}\right)$ of the floating strip in the computations (Fig. 2(c)). The voltage/current relationship imposed by symmetrical open-end capacitance at ports 5 and 6 is given by

$$
\bar{V}_{2}=Z_{\mathrm{c}} \overline{\bar{U}} \bar{I}_{2}
$$




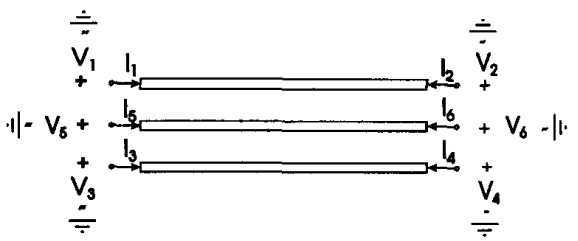

(a)

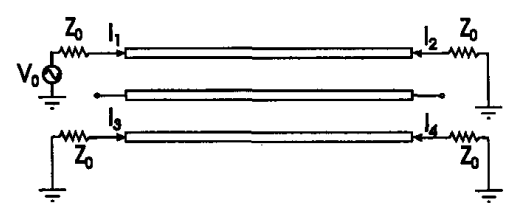

(b)

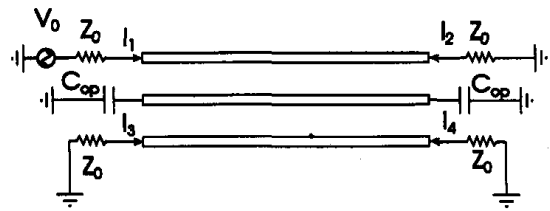

(c)

Fig. 2. (a) Circuit scheme of the three microstrip coupled line system. (b) The same circuit used as a four port coupler considering perfect open end at the floating conductor. (c) The same coupler including the open end capacitance at the floating conductor ends.

where $\overline{\bar{U}}$ is the $2 \times 2$ identity matrix and $Z_{c}$, the equivalent open-end impedance $Z_{c}=1 /\left(j \omega C_{\mathrm{op}}\right)$ due to the open-end capacitance. In this case, the impedance matrix relationship for the coupler becomes

$$
\bar{V}_{4}=\left\{\overline{\bar{Z}}_{4}+\overline{\bar{Z}}_{42}\left[Z_{c} \overline{\bar{U}}-\overline{\bar{Z}}_{2}\right]^{-1} \overline{\bar{Z}}_{24}\right\} \bar{I}_{4}
$$

Equation (7) shows that the effect of the open-end capacitance constitutes a correction term to the self-impedance matrix $\bar{Z}_{4}$. The $C_{\mathrm{op}}$ values used in this work have been calculated with the accurate spectral domain method in [11]. Approximate formulas for this parameter can be alternately used. Note that this effect cannot be considered using the even-odd mode model.

\section{COMPUTATION OF QuASI-STATIC PARAMETERS}

Solving (1) and (2) requires the knowledge of the p.u.l. capacitance matrices with $(\overline{\bar{C}})$ and without $\left(\overline{\bar{C}}_{v}=\left(1 / c^{2}\right) \overline{\bar{L}}^{-1}\right)$ dielectrics. Due to the symmetry of the problem, any of these matrices has the following form:

$$
\epsilon_{0} \overline{\bar{C}}^{-1}=\left[\begin{array}{lll}
a & b & c \\
b & d & b \\
c & b & a
\end{array}\right] \text {. }
$$

This section is intended as a brief explanation of the procedure to compute $a, b, c$ and $d$ in (8); they are required in three-mode model. Obviously, the even $\left(C_{e}\right)$ and odd $\left(C_{o}\right)$ modal capacitances are directly computed from them $\left(C_{e}^{-1}=a+c\right.$ and $\left.C_{o}^{-1}=a-c\right)$. Essentially, we followed the guidelines given in [7], also exploiting the symmetry of the problem. In this way, we compute the p.u.l. electrical energy $(U)$ stored in the structure for a suitable set of strip charge excitations. The quantity $U$ can be written in terms of the p.u.l. total free charge on each strip $\left(q_{1}, q_{2}, q_{3}\right)$ and the elements of (8)

$$
\epsilon_{0} U\left(q_{1}, q_{2}, q_{3}\right)=\frac{1}{2}\left(q_{1}, q_{2}, q_{3}\right) \cdot\left(\begin{array}{ccc}
a & b & c \\
b & d & b \\
c & b & a
\end{array}\right) \cdot\left(\begin{array}{l}
q_{1} \\
q_{2} \\
q_{3}
\end{array}\right) .
$$

By properly choosing the charge on each strip we can write

$$
\begin{aligned}
\epsilon_{0} U(1,0,-1) & =a-c \\
\epsilon_{0} U(1,0,1) & =a+c \\
\epsilon_{0} U(0,1,0) & =d / 2
\end{aligned}
$$

$$
\epsilon_{0} U(1,1,1)=\epsilon_{0} U(1,0,1)+\epsilon_{0} U(0,1,0)+2 b .
$$

The computation of the energy for the set of excitations in (10)-(13) then makes it possible to obtain the inverse of the capacitance matrix, both with and without dielectrics. This particular choice of excitations presents some interesting advantages. First, note that we consider one odd excitation and three even excitations. The plane $x=0$ can be then considered as either an electric or a magnetic wall, thus reducing the problem to the solution of the Laplace's equation in one of the halves of the structure ( $x>0$, for instance). The charge distribution on the strips- $\sigma_{i}(x) ; i=1,2,3$-for each excitation is expanded into the set of basis functions used in [8] (analysis of the boxed off-centered microstrip), i.e.,

$$
\sigma_{i}(x)=\sum_{p=1}^{N f_{i}} a_{i, p} \frac{2}{\pi w_{i}} \frac{T_{p}\left(x^{\prime}\right)}{\sqrt{1-x^{\prime 2}}} ; \quad x^{\prime}=\frac{x-d_{i}}{w_{i} / 2}
$$

where $T_{p}(\cdot)$ are the first kind Chebyshev polynomials; $w_{i}$, the width of the $i$ th strip and $d_{i}$, its central abscisa. Only even/odd functions are considered in the central strip for even/odd excitation. It is well known that the basis functions in (14) are especially appropriate for strip-like problems. The $a_{i, p}$ coefficients in (14) are computed using the Ritz-Galerkin technique in the spectral domain and the p.u.l. energy is computed from them [7]. The most time consuming step in the analysis is the evaluation of the spectral series defining some of the elements of the final linear equations system: those involving interactions between basis functions defined either on the same strip or on coplanar strips. Fortunately, thanks to the symmetry of the structure and excitations, this process was drastically accelerated using the quasi-closed form formulae in [8]. Extremely quick and accurate computer codes are generated this way.

It should be also mentioned that computations associated to even excitations are simultaneous and highly redundant. In conclusion, the overall computation time does not much differ from that necessary for the single microstrip case studied in [8]: typically a fraction of one second using a PC/386. It is remarkable that the computation of the three-mode model parameters requires no more effort than the computation of $C_{e}$ and $C_{o}$, due to existence of simple, useful relationships to carry out intermediate computations. Thus, the use of the 
TABLE I

Physical Description (Including Central Frequency Coupling) of a Number of Coupler Examples. Some Relevant Electrical Parameters of these Couplers are SHown IN TABle II.

\begin{tabular}{|c|c|c|c|c|c|c|c|c|c|}
\hline COUPLER C(dB) & $\begin{array}{c}w_{1} \\
(\mathrm{~mm})\end{array}$ & $\begin{array}{c}s \\
(\mathrm{~mm})\end{array}$ & $\begin{array}{c}w_{2} \\
(\mathrm{~mm})\end{array}$ & $\epsilon_{r}^{1}$ & $\begin{array}{c}h_{1} \\
(\mathrm{~mm})\end{array}$ & $\epsilon_{r}^{2}$ & $\begin{array}{c}h_{2} \\
(\mathrm{~mm})\end{array}$ & $i_{r}^{3}$ & $\begin{array}{c}h_{3} \\
(\mathrm{~mm})\end{array}$ \\
\hline ref. [3] (2.34) & 2.6 & 0.53 & 5.73 & 2.1 & 3.2 & 2.2 & 0.254 & air & $\infty$ \\
ref. [4] (2.66) & 0.54 & 0.5 & 1.5 & air & 5 & 2.1 & 0.051 & 2.1 & 0.787 \\
A1 (3.51) & 0.43 & 0.05 & 0.9 & air & $\infty$ & 2.52 & 0.135 & 10 & 1.27 \\
A2 (2.42) & 1.21 & 0.1 & 2.3 & air & $\infty$ & 2.52 & 0.135 & 2.56 & 1.524 \\
A3 (3.00) & 1.35 & 0.35 & 2.3 & air & $\infty$ & 2.56 & 1.524 & 2.56 & 1.524 \\
A4 (3.73) & 0.32 & 0.05 & 0.7 & air & $\infty$ & 10 & 0.635 & 10 & 1.27 \\
A5 (3.63) & 0.52 & 0.1 & 1.14 & air & $\infty$ & 2.56 & 0.135 & 10 & 1.524 \\
A6 (3.00) & 1.52 & 0.35 & 2.42 & 2.56 & 1.524 & 2.56 & 0.135 & air & $\infty$ \\
A7 (3.00) & 1.35 & 0.2 & 2.3 & 2.43 & 1.252 & 2.52 & 0.135 & air & $\infty$ \\
& & & & & & & & & \\
\hline B1 (3.41) & 1.2 & 0.1 & 2.5 & air & $\infty$ & 10 & 0.635 & 2.56 & 1.524 \\
B2 (3.17) & 1.48 & 0.1 & 3 & 2.56 & 1.524 & 10 & 0.635 & air & $\infty$ \\
B3 (4.83) & 0.89 & 0.1 & 1.88 & 2.43 & 0.49 & 10 & 0.635 & air & $\infty$ \\
B4 (5.87) & 0.53 & 0.1 & 1.16 & air & $\infty$ & 10 & 0.635 & 2.43 & 0.49 \\
B5 (4.84) & 2.35 & 0.2 & 2 & 2.55 & 1.524 & 10 & 1.270 & air & $\infty$ \\
& & & & & & & & \\
\hline
\end{tabular}

simple even-odd mode model is justified only as a preliminary step in the design process.

\section{VALIDITY OF THE TWO-MODE MODEL}

As previously stated, the structure under consideration should be analyzed as a three-conductor system supporting three independent quasi-TEM-type modes. However, experimental evidence shows that the even-odd mode theory yields correct results under certain circumstances. The purpose of this section is to provide an explanation for this fact.

We have analyzed a number of high coupling couplers, using the theories presented in section 2 in conjunction with the modal parameters given in the Appendix. The scattering parameters computed by means of the even-odd mode theory have been checked against those obtained with the three-mode model. Both theories yield similar results for some cases. However, large discrepancies have been found in some other cases. Table I shows the physical description of a number of $50 \Omega$ coupled sections with various coupling levels. Table II includes the values of certain relevant electrical parameters of couplers in Table I. It includes four columns containing the following parameters:

$$
\begin{aligned}
& c_{1}=\left(C_{11}+C_{13}\right) /\left(C_{11}^{v}+C_{13}^{v}\right) \\
& p_{1}=\left(P_{11}^{v}+P_{13}^{v}\right) /\left(P_{11}+P_{13}\right) \\
& c_{2}=C_{22} / C_{22}^{v} \\
& p_{2}=P_{22}^{v} / P_{22}
\end{aligned}
$$

where $C_{i j}$ and $C_{i j}^{v}$ are the elements of the capacitance matrices with and without dielectrics, respectively; $P_{i j}$ and $P_{i j}^{v}$ are the elements of the $\overline{\bar{P}}=\overline{\bar{C}}^{-1}$ and $\overline{\bar{P}}_{v}=\overline{\bar{C}}_{v}^{-1}$ matrices, respectively.

The coupler designs in these tables have been arranged in two different groups. Group A corresponds to cases for which the even-odd mode analysis and the three-mode analysis provide essentially the same results. Group B contains those cases which obtain significant discrepancies between these models. Table II shows that, for couplers in group A, either
TABLE II

Relevant Electrical Parameters for Couplers in Table

\begin{tabular}{|c|c|c|c|c|c|c|c|c|}
\hline COUPLER & $\epsilon_{b}$ & $\epsilon_{\mathrm{c}}$ & $c_{1}$ & $c_{2}$ & $p_{1}$ & $p_{2}$ & $R_{I b}$ & $R_{I \mathrm{c}}$ \\
\hline [3] & 1.749 & 2.155 & 2.097 & 1.797 & 1.749 & 2.154 & 0.12 & -2.21 \\
\hline [4] & 2.071 & 1.747 & 2.071 & 1.747 & 1.798 & 2.013 & -1.79 & -33.65 \\
\hline AI & 6.702 & 2.348 & 3.777 & 4.165 & 6.673 & 2.358 & -0.16 & -2.39 \\
\hline A2 & 2.479 & 2.052 & 2.478 & 2.053 & 2.137 & 2.381 & -1.70 & -14.85 \\
\hline A3 & 2.463 & 2.093 & 2.459 & 2.093 & 2.155 & 2.388 & -1.70 & 102.99 \\
\hline A4 & 9.636 & 6.262 & 9.614 & 6.276 & 8.699 & 6.937 & -0.84 & -20.65 \\
\hline A5 & 6.693 & 2.419 & 3.645 & 4.442 & 6.665 & 2.429 & -0.18 & -2.33 \\
\hline A6 & 2.076 & 2.517 & 2.421 & 2.158 & 2.076 & 2.516 & -0.09 & -2.23 \\
\hline A7 & 2.006 & 2.486 & 2.394 & 2.083 & 2.006 & 2.486 & 0.09 & -2.22 \\
\hline B1 & 2.797 & 8.356 & 7.149 & 3.269 & 3.014 & 7.756 & 1.29 & -1.96 \\
\hline B2 & 2.754 & 8.491 & 7.945 & 2.943 & 3.222 & 7.257 & 3.29 & -2.04 \\
\hline B3 & 2.900 & 7.836 & 7.360 & 3.090 & 4.050 & 5.605 & 5.58 & -2.01 \\
\hline B4 & 3.094 & 7.487 & 5.565 & 4.162 & 3.374 & 6.866 & 0.92 & +1.99 \\
\hline B5 & 3.110 & 7.538 & 6.339 & 3.698 & 3.630 & 6.459 & 1.77 & -1.89 \\
\hline
\end{tabular}
I. Couplers IN Group A CAN Be INDISTINCTLY ANALYZED USINO THE EVEN-OdD OR THE THREE-MOdE APPROACHES. COUPLERS in Group B Must be ANalyzed Using the Three-Mode Model.

$\left|R_{I 1}\right| \gg\left|R_{I 2}\right|$ or $\left|R_{I 2}\right| \gg\left|R_{I 1}\right|$. This implies that if one of the hot strips is excited, most of the power is carried by the odd mode and only one of the two even modes. It is then expected that the coupler response can be computed by assuming twomode operation. This situation corresponds to a certain degree of decoupling between the two hot strips and the floating strip.

Note that, if we consider a two conductor coupled line (i.e., the floating conductor is suppressed), $c_{1}$ would equal $p_{1}$, the conventional even mode propagation constant in the two-mode model. These parameters differ due to the presence of the floating conductor. We can see from Table II that either $c_{1}$ and $c_{2}$ or $p_{1}$ and $p_{2}$ are very close to the even mode eigenvalues of the three line system $\left(\epsilon_{b}, \epsilon_{c}\right)$ for couplers in group A. In any of these cases, the conventional even/odd mode theory may be used confidently. None of these coincidences occur for couplers in group B. Thus, a complete three-mode analysis should be used in this case. Consequently, we now have a criterion to determine whether a given structure can or cannot be analyzed using the even-odd mode model. Some experimental evidence of the correctness of this conclusion are included in the section "results."

\section{Full-Wave Computations}

Dispersion in this structure is expected to be negligible for frequencies in the order of a few gigahertzs (for usual substrate thicknesses and permittivities). In any case, this statement should be proven by comparison with full-wave computations. We have implemented a full-wave spectral domain analysis to ensure that dispersion is actually negligible. We used the spectral domain formulation in [12] to compute the frequency dependent modal propagation constants, i.e., the parameters in the Appendix as a function of the operating frequency. Since the mathematics in [12] were developed for coplanar strips, we introduced some minor modifications to account for non-coplanar strips. In order to do this, we obtained the closedform spectral domain Green's dyadic relating electric fields and surface currents at the two metallized interfaces. 
The basis functions used in this work to approximate the current distributions- $j_{z}(x), j_{x}(x)$-on the strips are

$$
\begin{aligned}
& j_{i, z}(x)=\sum_{p=1}^{n t f_{i z}} a_{i, p}^{z} \frac{2}{\pi w_{i}} \frac{T_{p}\left(x^{\prime}\right)}{\sqrt{1-x^{\prime 2}}} \\
& j_{i, x}(x)=\sum_{q=1}^{n t f_{i x}} a_{i, q}^{x} \frac{2}{\pi w_{i}}(q+1) U_{q}\left(x^{\prime}\right) \sqrt{1-x^{\prime 2}}
\end{aligned}
$$

where $U_{q}(\cdot)$ stands for the second order Chebyshev polynomials ( $p$ and $q$ are integers), and $x^{\prime}$ has been already defined. These functions are especially suitable due to the nature of the singularities at the strip edges. Once again, the numerical series involved in the spectral analysis can be quickly summed using the same procedure as in quasi-TEM computations. Nevertheless, the full-wave analysis is much more time consuming than quasi-TEM analysis, and should be avoided if dispersion is not important.

The propagation constants and current eigenvectors, $\bar{I}_{p}$ are simultaneously obtained solving the eigenvalue problem for the modal propagation constants $\left(\beta_{p}\right.$ 's, $\left.p=a, b, c\right)$. The voltage eigenvectors, $\bar{V}_{p}(p=a, b, c)$ must be then computed from the known $\bar{I}_{p}$ eigencurrents and the power flowing through the structure for each mode, $P_{p}$ (for a detailed discussion on the problem of the definition and computation of impedance parameters refer to [13]):

$$
P_{p}=\frac{1}{2} \bar{V}_{p}^{t} \cdot \bar{I}_{p} \quad, p=a, b, c .
$$

The modal power flux, $P_{p}$, can be written in terms of the surface current for the $p$ th mode. These computations are carried out by extending the technique reported in [13] to noncoplanar structures. Since this is not the aim of this paper, we will not go into the details of these computations.

In sum, we may state that an efficient full-wave analysis has been carried out for the structure in Fig. 1 to ensure that frequency dispersion does not affect our design. If the dispersion is significant, we may use these frequency dependent parameters to correct the design, based on quasi-TEM simulation.

\section{THEORETICAL AND EXPERIMENTAL RESUlTS}

Before generating design data we exhaustively checked performance of the FORTRAN computer codes to obtain both quasi-TEM and dynamic parameters. Convergence tests were performed to ensure accuracy and reliability. We first considered the convergence of the spectral series defining the entries of the Galerkin matrix. Thanks to the use of asymptotic extraction techniques we obtain virtually exact results for those series. Discussions about the convergence and computation speed in [8] also apply here. In addition, we studied the convergence of the results against the number of basis functions. A typical convergence pattern for the four distinct parameters in (8) is shown in Fig. 3. As a general conclusion we may state that only a few (two or three) basis functions on each strip must be retained to obtain very accurate data. A few more basis functions should be added if the strip separation is extremely narrow or in the case of

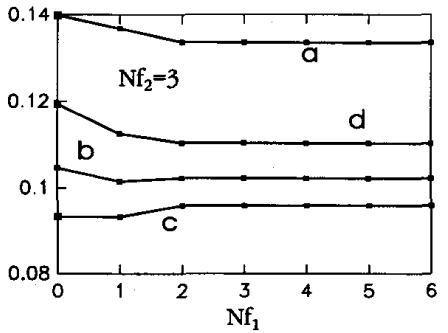

(a)

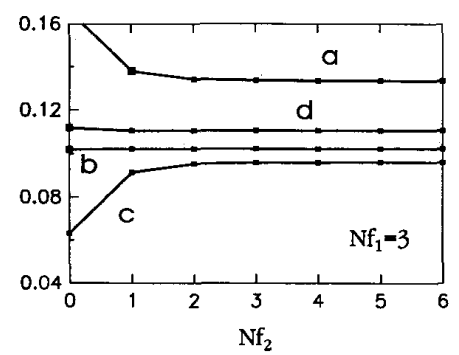

(b)

Fig. 3. Typical convergence pattern for the elements of $\epsilon_{o} \overline{\bar{C}}^{-1}$ matrix: $a, b, c$ and $d$ (a) versus the number of basis functions on the coplanar strips $\left(N_{f 1}\right)$ and (b) versus the number of basis functions on the floating strip $\left(N_{f 2}\right)$. These curves correspond to coupler A7 in Table I.

very thin upper substrate. Typical CPU time for quasi-TEM computations is well below one second using a PC/386 with a math coprocessor. A similar conclusion also apply to dynamic computations.

In Fig. 4 we show the frequency dependent parameters of the coupler called B3 in Table I. It can be seen that dispersion is not very important below $5 \mathrm{GHz}$. The scattering parameters computed with this frequency dependent data do not differ significantly from those computed using quasi-TEM data. In Fig. 5 we show theoretical scattering parameters for coupler B3 calculated with a quasi-TEM model. Square points represent dynamic data. It is obvious that the difference does not justify the use of the full-wave model. This is true for all frequencies, substrates and dimensions considered in the examples in this paper. Thus, the quasi-TEM analysis was finally used to generate the theoretical scattering parameters contained in this work. Due to the deterministic nature of the quasi-TEM analysis (no iterative eigenvalue searching is needed as in full-wave analysis) it has been easy to write an optimization program which provides the dimensions of the coupler for a given substrate configuration and target coupling. The process typically converges in just a few. iterations. This program considered that $w_{2}=2 w_{1}+s$, since this choice obtains maximum coupling [4].

Once the computer codes were considered reliable, we tried to reproduce the theoretical and experimental results reported in [3], [4]. Our theoretical results agree quite well with theoretical and experimental data reported in those papers. In addition, the even-odd mode theory and the three-mode model provide almost identical results for those cases. Moreover, we fabricated two different couplers to validate our theoretical 


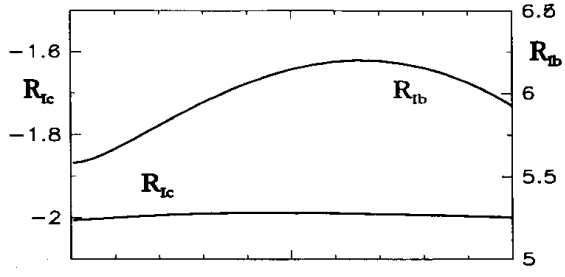

(a)

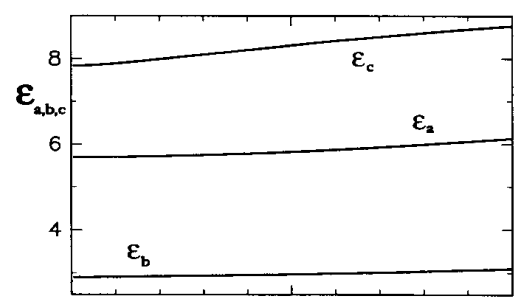

(b)

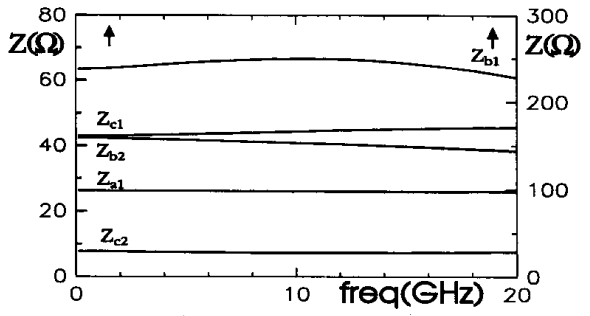

(c)

Fig. 4. (a) Mode numbers $\left(R_{I(b, c)}\right)$, (b) normalized effective dielectric constants $\left(\epsilon_{(a, b, c)}\right)$, and (c) modal impedances $\left(Z_{a 1}, Z_{b 1}, Z_{b 2}, Z_{c 1}, Z_{c 2}\right)$ as a function of frequency for the coupler $B 3$ in Table $I$.

conclusions. One belongs to group A (A7), and the other to group B (B3) (see Tables I and II). The scattering parameters were measured with an HP-8510 vector network analyzer. Measured data obviously include unavoidable experimental errors due to connectors mismatching and other error sources. No adjustment was performed after the fabrication process. Fig. 6 shows the experimental and theoretical results for the coupler A7 in Table I. The agreement between theoretical and experimental data is reasonably good.

In this case, the scattering parameters predicted by the even-odd mode theory and the three-mode theory are indistinguishable on the curves. This is not true for the couplers belonging to group B in Table I. For instance, we have also fabricated and measured coupler B3 in that Table. Fig. 7 shows the theoretical response predicted by the even-odd mode theory and the three-mode theory for this coupler, including a number of experimental points. It is clear that a threemode model must be used to predict the performance of this coupler. It should be emphasized that the effect of the open end capacitance has been considered in the theoretical simulation $\left(C_{\mathrm{op}}=0.120 \mathrm{pF}\right)$. In order to have a feeling on the effect of this capacitance, we have represented in Fig. 8 the coupling computed with and without the open end capacitance. Note that the resonant behavior of the scattering parameters around the $3 \mathrm{GHz}$ frequency point is associated to the three-
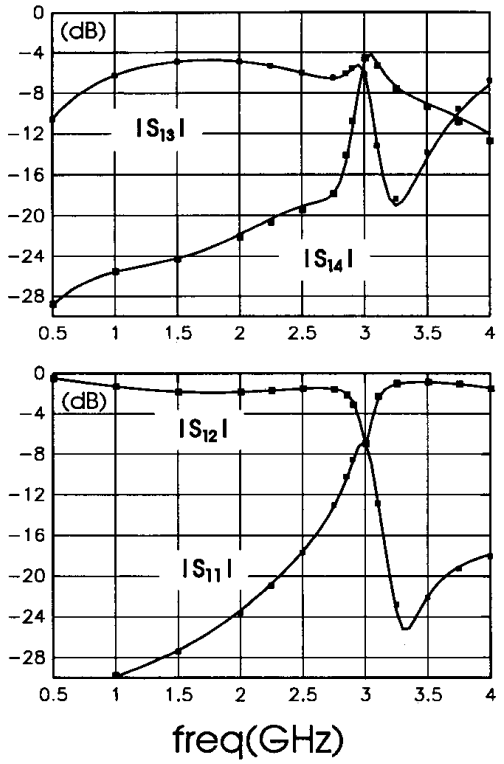

Fig. 5. Theoretical $S$-parameters for the coupler B3 (Table I) computed using quasi-TEM (solid line) and full-wave (square points) models.

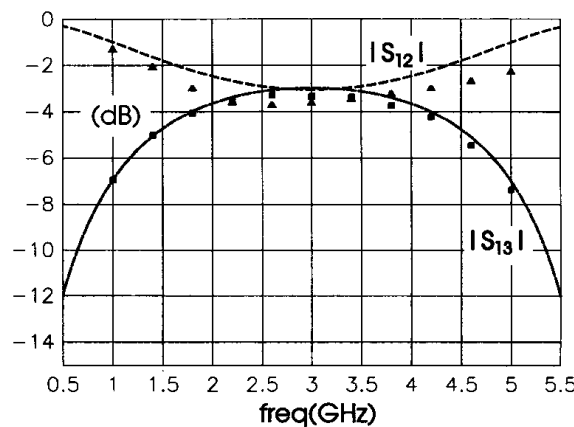

Fig. 6. $\left|S_{12}\right|$ and $\left|S_{13}\right|$ scattering parameters for the coupler A7 in Table I (the coupling length is $17.26 \mathrm{~mm}$ ). Two and three-mode theories yield the same theoretical results (using both quasi-TEM and dynamic data). Squares and triangles correspond to measured values.

mode operation, rather than to the open end capacitance. The effect of the open end capacitance is to slight shift that resonant frequency. We have observed that this shift is more significant for wide strips. In contrast, the two-mode model does not account at all for the response of the coupler around that critical frequency. Moreover, we also concluded from theoretical simulations that even an "even-odd analyzable" structure should be analyzed with the three-mode model if the open end capacitance is very high.

The agreement between theoretical and experimental results for return loss and isolation is not so good. This is not surprising, since these parameters are affected by a number of factors (discontinuities, measuring errors) which are not taken into account. Nevertheless, the three-mode theory also accounts for the degradation of matching and isolation around $3 \mathrm{GHz}$ (and not in the two-mode one). 


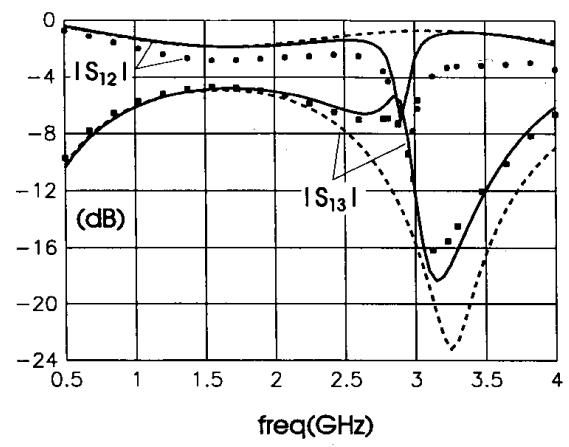

(a)

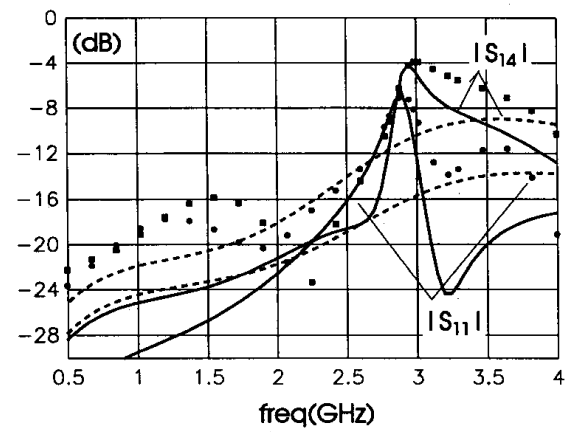

(b)

Fig. 7. (a) $\left|S_{12}\right|$ and $\left|S_{13}\right|$ for the coupler B3 in Table I (the coupling length is $20 \mathrm{~mm}$ ). (b) $\left|S_{11}\right|$ and $\left|S_{14}\right|$ for the same coupler. Solid lines are theoretical results obtained with the three-mode model. Dashed lines correspond to the even-odd model. Squares and circles are measured values.

\section{CONCLUSION}

This paper presents the study of a semi re-entrant microstrip section suitable for tight coupling applications. Electrical parameters of the coupled section have been computed using efficient quasi-TEM and full-wave analysis. Quasi-analytical computations have been incorporated to speed up the program execution. Quasi-TEM results have proven to be useful for low frequency operation and they may be computed with very high accuracy and very short CPU time. Thanks to this, automatic design of the coupler section can be carried out in, at most, a few seconds using a personal computer.

The scattering parameters have been computed based on the conventional even-odd mode theory and a more rigorous three-mode model. The conditions under which the simplified even-odd theory works well enough have been derived. A couple of couplers have been fabricated and measured to verify the correctness of the theoretical results. Experimental data confirm the need to use the three-mode model to accurately predict the parasitic resonant behaviour arising in some coupler realizations using the structure analyzed in this paper.

\section{APPENDIX}

The structure analyzed in this paper is a nonhomogeneous symmetrical three conductor transmission line system, whose modal parameters were reported in [5]. These parameters are

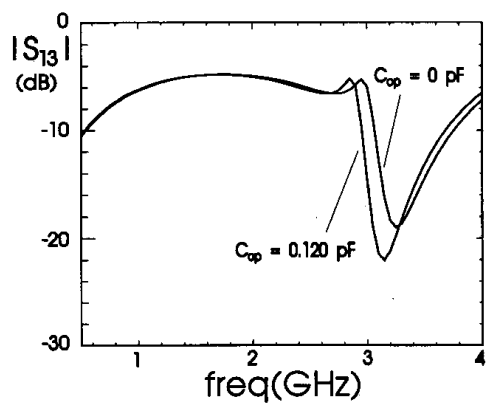

Fig. 8. $\left|S_{13}\right|$ for coupler B3 with and without open end capacitance.

expressed in terms of the elements of the dimensionless matrix $c^{2} \overline{\bar{L}} \cdot \overline{\bar{C}}$ matrix ( $c$ being the light speed in vacuum). Let us reproduce the formulas from that paper which are relevant to our analysis

$$
c^{2} \quad \overline{\bar{L}} \cdot \overline{\bar{C}}=\left[\begin{array}{lll}
A & B & C \\
D & E & D \\
C & B & A
\end{array}\right] .
$$

The eigenvalues of (22), that is, the modal effective dielectric constants (normalized to $\epsilon_{0}$ ), are

$\epsilon_{a}=A-C ; \epsilon_{b, c}=\frac{A+C+E}{2} \pm 1 / 2 \sqrt{(A+C-E)^{2}+8 D B}$

where $\epsilon_{a}$ corresponds to the odd mode and $\epsilon_{b, c}$, to the even modes. The associated voltage and current eigenvectors may be arranged into two matrices

$$
\begin{gathered}
\overline{\bar{M}}_{V}=\left(\bar{V}_{a}, \bar{V}_{b}, \bar{V}_{c}\right)=\left[\begin{array}{ccc}
1 & 1 & 1 \\
0 & R_{V b} & R_{V c} \\
-1 & 1 & 1
\end{array}\right] \\
\overline{\bar{M}}_{I}=\left(\bar{I}_{a}, \bar{I}_{b}, \bar{I}_{c}\right)=\left[\begin{array}{ccc}
1 & 1 & 1 \\
0 & R_{I b} & R_{I c} \\
-1 & 1 & 1
\end{array}\right]
\end{gathered}
$$

where

$$
\begin{gathered}
R_{V b, c}=-\frac{A+C-E}{2 B} \pm \sqrt{\left(\frac{A+C-E}{2 B}\right)^{2}+2 \frac{D}{B}} \\
R_{I b, c}=-2 / R_{V c, b} .
\end{gathered}
$$

The $R_{V x}$ and $R_{I x}(x=b, c)$ parameters are the so-called voltage and current mode numbers for the even-type modes supported by the structure. The characteristic impedances of the three lines for the three normal modes are

$$
\begin{aligned}
Z_{a 1}=Z_{a 3} & =c\left(L_{11}-L_{13}\right) / \sqrt{\epsilon_{a}} \\
Z_{b 1}=Z_{b 3} & =c\left(L_{11}+L_{13}+R_{I b L_{12}}\right) / \sqrt{\epsilon_{b}} \\
Z_{c 1}=Z_{c 3} & =c\left(L_{11}+L_{13}+R_{I c} L_{12}\right) / \sqrt{\epsilon_{c}} \\
Z_{b 2} & =c\left(R_{I b} L_{22}+2 L_{12}\right) /\left(R_{I b} \sqrt{\epsilon_{b}}\right) \\
Z_{c 2} & =c\left(R_{I c} L_{22}+2 L_{12}\right) /\left(R_{I c} \sqrt{\epsilon_{c}}\right) .
\end{aligned}
$$

The parameters in this Appendix fully characterize the threeline system from a circuit theory point of view. 


\section{REFERENCES}

[1] R. K. Hoffmann, Handbook of Microwave Integrated Circuits. Norwood, MA: Artech House, 1987

[2] J. A. G. Malherbe and I. E. Losch, "Directional couplers using semireentrant coupled lines," Microwave J., pp. 121-128, Nov. 1987.

[3] M. Karam and Y. Demers, "Theory and design of wide-band $3 \mathrm{~dB}$ suspended microstrip directional couplers with floating conductor," Microwave Optical Tech. Lett., vol. 3, pp. 181-191, May, 1990.

[4] M. Nakajima and E. Yamashita, "A quasi-TEM design method for 3 $\mathrm{dB}$ hybrid couplers using semi-reentrant coupling section," IEEE Trans. Microwave Theory Tech., vol. 38, pp. 1731-1733, Nov. 1990.

[5] V. K. Tripathi, "On the analysis symmetrical three-line microstrip circuits," IEEE Trans. Microwave Theory Tech., vol. MTT-25, pp. 726-729, Sep. 1977.

[6] characteristically terminated three-line structures in an inhomogeneous medium," IEEE Trans. Microwave Theory Tech., vol. MTT-29, pp. 22-26, Jan. 1981.

[7] F. Medina and M. Horno, "Capacitance and inductance matrices for multistrip structures in multilayered anisotropic dielectrics," IEEE Trans. Microwave Theory Tech., vol. MTT-35, pp. 1002-1008, Nov. 1987.

[8] F. Medina and M. Horno, "Quasi-analytical static solution of the boxed microstrip line embedded in a layered medium," IEEE Trans. Microwave Theory Tech., vol. 40, pp. 1748-1756, Sept. 1992.

[9] L. S. Napoli and J. J. Hughes, "Characteristics of coupled microstrip lines," RCA Rev., pp. 479-498, Sep. 1970.

[10] S. Frankel, Multiconductor Transmission Line Analysis Norwood MA: Artech House, 1977.

[11] J. Martel, R. R. Boix, and M. Horno, "Static analysis of microstrip discontinuities using the excess charge density in the spectral domain," IEEE Trans. Microwave Theory Tech., vol. 39, pp. 1623-1631, Sept. 1991.

[12] F. Medina, M. Horno, and H. Baudrand, "Generalized spectral analysis of planar lines on layered media including uniaxial and biaxial dielectric substrates," IEEE Trans. Microwave Theory Tech., vol. 37, pp. 504-511, Mar. 1989.

[13] G. Cano, F. Medina, and M. Horno, "Efficient spectral domain analysis of generalized multistrip lines in stratified media including thin, anisotropic, and lossy substrates," IEEE Trans. Microwave Theory Tech. vol. 40, pp. 217-227, Feb. 1992.

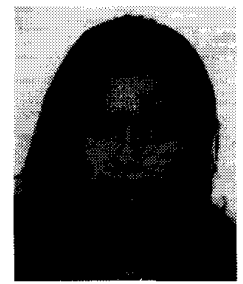

Fátima Masot was born on January 29, 1964 in Badajoz, Spain. She received the Licenciada degree in Physics from the University of Seville, Spain, in 1987.

She had a scholarship of the Spanish Government and is currently Assistant Professor. Her research interests focus on the analysis of directional couplers and filters and other multiconductor line circuits.

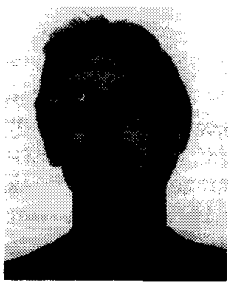

Francisco Medina was born in Puerto Real, Cdiz, Spain, in November, 1960 . He received the Licenciado degree in September 1983 and the Doctor degree in 1987, both in Physics, from the University of Seville, Spain.

He is currently Associate Professor of Electricity and Magnetism in the Department of Electronics and Electromagnetics, University of Seville. His research deals mainly with analytical and numerical methods for planar structures and the analysis of multiconductor line systems. He has been a member of the Technical Programme Committee of the 23rd European Microwave Conference (Madrid, Spain, Sept. 1993).

Manuel Horno (M'75) for photograph and biography, see page 432 of the March issue of this TRANSACTIONS. 